\title{
VALUE OF DIFFERENT ECHOCARDIOGRAPHIC MODALITIES IN THE ASSESSMENT OF LEFT VENTRICULAR HYPERTROPHY IN HYPERTENSIVE PATIENTS WITH TYPE II DIABETES MELLITUS
}

\author{
By
Hossam Farid Hamada, Ahmad Kamal Motawea, Mohammad Saad El- Gammal and Mohammad Ismail Al-Deftar \\ Department of Cardiology, Faculty of Medicine, Al-Azhar University, (Cairo) \\ Corresponding author: Hossam Farid Hamada;
}

Phone: 01221617344; E-mail: hossamfarid@hotmail.com

\begin{abstract}
Background: Left ventricular hypertrophy $(\mathrm{LVH})$ is the response of myocytes to various stimuli leading to myocytes' hypertrophy, which occurs as a compensatory response to increased after-load. It is defined as an increase in LV mass assessed by postmortem measurements, electrocardiographic (ECG), echocardiographic and cardiovascular magnetic resonance (CMR) criteria.

Objective: To investigate the reproducibility of different echocardiographic modalities in the assessment of LVH in hypertensive patients and patients with type II DM in comparison to the golden standard CMR measurements.

Patients and Methods: We prospectively investigated 40 patients with $\mathrm{LVH}, 20$ patients being hypertensive and diabetic, 20 patients being diabetic only and 20 healthy control subjects (normal ventricular function and ECG and no cardiac risk factors). In addition to clinical and conventional echocardiographic parameters, LV mass and volumes were analyzed.

Results: The comparison between new echocardiographic techniques and CMR showed that the assessments of LV volumes/LVEF by echocardiography and CMR have good correlations. However, the inter-technique agreement of absolute LV volumes revealed considerable differences, with significant underestimation of volumes and LVEF with respect to CMR. The LV mass was less in patients assessed by different algorithms of 2D echo rather than 3D echo and CMR, correlated positively with EDV and ESV.

Conclusion: 3D echocardiography attains more solid results in patients diagnosed having LVH by 2D echocardiography. CMR would be preferable for research and specific clinical conditions requiring higher accuracy and reproducibility.
\end{abstract}

Keywords: Echocardiographic, LVH, Hypertensive Patients, Type II Diabetes Mellitus.

\section{INTRODUCTION}

Left ventricular hypertrophy $(\mathrm{LVH})$ is the response of myocytes to various stimuli leading to myocytes' hypertrophy, which occurs as a compensatory response to increased afterload. It is defined as an increase in LV mass, assessed by postmortem measurements, electrocardiographic (ECG), echocardiographic and Cardiovascular Magnetic Resonance (CMR) criteria. Early echocardiographic studies defined 
LVH as an absolute LV mass (LVM) exceeding $250 \mathrm{~g}$ (Drazner et al., 2011).

Regression of LVH reduces the risk of stroke, myocardial infarction and all-cause mortality. There are two main patterns of LVH: a) concentric and b) eccentric LVH. Concentric LVH is considered, when LV mass increases by wall thickening in response to pressure overload, as often in middle aged and elderly patients, is associated with lower cardiac output and predicts poor prognosis. There is a pathway from hypertension to concentric LVH without focal scar (Yoneyama et al., 2012), hypertension to concentric LVH with focal scar (Ambale-Venkatesh et al., 2014), concentric remodeling with myocardial infarction assessed by replacement fibrosis (Turkbey et al., 2015), and concentric LVH with symptomatic vascular events and heart failure either with replacement scar (Schelbert et al., 2012) or without (Chahal et al., 2015).

Diastolic dysfunction and/or heart failure with preserved ejection fraction (HFpEF), due to remodeling of the extracellular matrix and increase in LV filling pressures, are common in concentric LVH (Liu et al., 2013). In eccentric $\mathrm{LVH}$, there is an increase in $\mathrm{LV}$ mass without increased concentricity and is associated with higher cardiac output (Chahal et al., 2015). It has not been fully clarified why patients develop a specific LVH pattern, as a response to different stimuli. Factors such as pressure, volume overload, ethnicity, gender, obesity and plasma renin levels, all seems to play a role (Drazner, 2011).

Various non-invasive techniques have been used to elucidate the pattern of $\mathrm{LVH}$.
ECG and echocardiography were for many years the only techniques for evaluation of LVH. Although ECG measures of LVH were associated with cardiovascular disease risk in the Framingham study, the ECG evaluation of LVH lacks sensitivity and specificity, particularly in young male patients (Bratincsák et al., 2015).

Discrepancy documented in diagnostic performance and agreement on predictive ability suggests that LVH by ECG and LVH by CMR are likely to be two distinct phenotypes (Bacharova et al., 2015).

CMR, due to its excellent reproducibility, unrestricted field of view and non-invasive, non-radiating tissue characterization, became a powerful player for early diagnosis and treatment assessment of LVH and gender-specific values according to age and body surface area have been already published. The comparison between new echocardiographic techniques and CMR showed that the assessment of $\mathrm{LV}$ volumes/LVEF by echocardiography and CMR have good correlations. However, the inter-technique agreement of absolute $\mathrm{LV}$ volumes revealed considerable differences, with significant underestimation of volumes and LVEF with respect to CMR (Aurich et al., 2014).

Another study evaluating if LVM by real-time, 3-dimensional echocardiography (RT-3DE) corresponded to CMR in patients with LVH, showed that LVM by RT-3DE correlated with that determined by CMR better than that determined by $2 \mathrm{DE}$, which means that RT-3DE can overcome some of the disadvantages of $2 \mathrm{DE}$ in the evaluation of LVM. However, another 
study, evaluating the accuracy of LVM calculation using new echocardiographic techniques in comparison with CMR in ischemic (IC) and nonischemic cardiomyopathy (non-IC), documented that although more accurate and reliable echocardiographic measurement of LVM was achieved by 3DE, underestimation and variability remained challenges in IC (Kusunose et al., 2013).

Another study, evaluating 40 patients by echocardiography using 4 imaging modalities (M-mode fundamental imaging [FI], M-mode harmonic imaging [HI], two-dimensional [2D] FI and 2D HI) and CMR, showed that $\mathrm{HI}$ overestimates LVM, compared with FI and CMR leading to overestimation of prevalence of LVH in hypertensive patients. HI improves inter-observer reproducibility of LVM measurements, compared with FI, leading to a significant decrease in the number of patients required for clinical trials of LVM regression. Finally, the accuracy of LVM measurements by echocardiography is affected by LV geometry (Park et al., 2014).

Cardiac magnetic resonance imaging (CMR) is an accurate and reliable means of evaluating cardiac morphology, and therefore very well suited for identifying and characterizing patients with various manifestations of left ventricular hypertrophy (LVH) (Brouwer et al., 2011).

Within the latest 10 years, research in $\mathrm{LVH}$ as cardiac target organ damage has uncovered its prognostic importance. Consequently, LV mass should be accurately calculated as mass size may have important clinical implications (Westenberg et al., 2010).
Brumback et al. (2016) sought for new accurate indices of LV mass. The main purpose of their study was to develop allometric indices for LV mass measured by CMR and to compare estimates of the prevalence and predictive value of $\mathrm{LVH}$.

In this study we aim to evaluate the effect of type II diabetes mellitus in hypertensive patients on LV mass index and geometry by conventional echocardiography in comparison with CMR.

\section{PATIENTS AND METHODS}

This study included 60 Patients referred to the echocardiography unit of Al-Hussein University hospital for conventional echocardiographic assessment in the period from October 2017 to October 2019 and were divided into three groups. Forty patients were found out to have LVH as documented by ECG and echocardiographic criteria, according to the diagnostic criteria of American Society of Echocardiography 2017. Twenty patients had the clinical diagnosis of hypertension made according to AHA guidelines 2017, and on medical treatment. Twenty patients were diabetics, diagnosed according to ADA guidelines 2015 with documented evidence of LVH and on medical treatment; another twenty subjects were taken as a control group, (normal ventricular dimensions and function and normal ECG with no history of cardiac disease).

\section{Exclusion criteria:}

All patients with congenital heart defects, valvular heart disease, atrial fibrillation, flutter or other arrhythmias, infectous disorders, malignant tumors, as well as patients with evidence of any type 
of pulmonary hypertension, chronic kidney disease, glycogen storage diseases and other hereditary disorders were excluded from the study.

All patients were studied along the following scheme:

A. Informed consent taken from all patients.

B. Complete history taking: highlighting onset, duration and complications of both hypertension and diabetes mellitus and medications received.

C. Clinical examination.

Full clinical examination was carried out on every patient with special emphasis on the following data: (1) Pulse: rate and rhythm, (2) Blood pressure, (3) Height in meters, (4) Chest and heart examination for heart sounds, additional heart sounds and murmur and the back for lung crackles.

D. Laboratory investigations: Including fasting and post prandial blood sugar, kidney functions tests and $\mathrm{HbAlc}$.

E. Resting 12 lead Electrocardiography: Resting standard 12-leads electrocardiogram searching for rate, rhythm, and chamber hypertrophy. Chamber hypertrophy was concluded according to Sokolov-Lyon criteria.

\section{F. Transthoracic Echocardiographic Examination (ASE 2015)}

Echocardiographic examination to all patients was done in the left lateral decubitus position between 20 and 45 degrees using Phillips i3 device with 3.0 $\mathrm{MHz}$ phased array transducer. Images were acquired from the standard views (parasternal long-axis, parasternal short axis at the mid cavity level, apical fourchamber and apical five-chamber).

The transducer was placed at the third to fifth left inter-costal space and sweeps were made from the aortic root to the mitral valve and toward the apex of the left ventricle.

\section{The following measurements were taken:}

The end-diastolic left ventricular posterior wall thickness (LVPWd), interventricular septal thickness (IVS,), and left ventricular internal dimension (LVIDd) were measured at the level of the chordae of the mitral valve at the peak of the $\mathrm{R}$ wave of the electrocardiogram using standard methods. The standard measurement convention includes the thickness of the right and left septal endocardial echoes in the IVSd and includes the posterior wall endocardial echoes in the LVPWd.

Normal left ventricular end-diastolic dimensions were considered to be the following: Left ventricular posterior wall $(\mathrm{LVPWd})=6-11 \mathrm{~mm}$, inter-ventricular septal thickness $($ IVSd) $=6-11 \mathrm{~mm}$, left ventricular internal dimension $($ LVIDd $)=$ $35-57 \mathrm{~mm}$.

Left ventricular mass was estimated from measurements using the standard convention as follows: Mass $(\mathrm{g})=0.77 \times$ 10-3 $\times$ [(LVIDd + LVPWd + IVSd $) 3-$ (LVIDd)3] + 2.4 where the various dimensions are given in millimeters.

Left ventricular mass was also estimated from measurements using the Penn convention. Measurements with the Penn convention excluded the right and left septal endocardial echo thickness from the IVSd and this convention 
excludes the posterior wall endocardial echo thickness from the LVPWd. Left septal endocardial echo thickness and posterior wall endocardial echo thickness are thus included in the LVIDd by this method. Using the Penn convention, left ventricular mass is estimated as: Mass ( $\mathrm{g}$ ) $=1.04[($ LVIDd + LVPWd + IVSd $) 3-$ (LVIDd)3] - 13.6 where the dimensions are given in millimeters.

These equations permit an estimate of left ventricular mass by assuming that the ventricle is ellipsoidal during end diastole. The internal volume of the ventricle is subtracted from the external volume, which gives the volume of the ventricular muscle. Mass is estimated from the specific gravity of ventricular muscle, which is assumed to be $1.05 \mathrm{~g} / \mathrm{cm} 3$.

\section{G. CMR measurements.}

A set of contiguous short axis slices covering the entire LV from the atrioventricular ring down to the apex, acquired from a cine sequence. $\mathrm{A}$ combination of body matrix/torso radio frequency coils was used for the acquisition, using a 2D cardiac gated pulse sequence. Ideally, images were acquired at resting lung volume. Myocardial volume is the area occupied between the endocardial and epicardial border multiplied by the interslice distance. By convention, LVM was measured at end diastole. Similar to echocardiography, LVM is the product of this volume and the density of the myocardium.

\section{Statistical analysis:}

Data was analyzed using Statistical program for Social Science (SPSS) version 25.0 for Windows. Quantitative data was expressed as mean \pm standard deviation (SD). Qualitative data was expressed as frequency and percentage. Probability (p-value): p-values $<0.05$ was considered significant, p-values $<0.001$ was considered as highly significant and p-values $>0.05$ was considered insignificant. 


\section{RESULTS}

There was no statistically significant difference between the three groups and 2D echocardiographic data regarding 2D LVEF, 2D EDV, 2D ESV, 2D SV while there was highly statistically significant difference as regard 2D IVSd, 2D PWd, 2D LV mass (Table 1).

Table (1): Comparison between the studied groups regarding the 2D echocardiographic data

\begin{tabular}{|c|c|c|c|c|c|c|}
\hline \multirow{2}{*}{\multicolumn{2}{|c|}{ Parameters $\quad$ Groups }} & \multirow{2}{*}{\multicolumn{2}{|c|}{$\begin{array}{c}\text { Control group } \\
\text { No. }=\mathbf{2 0}\end{array}$}} & \multirow{2}{*}{$\begin{array}{c}\text { HTN group } \\
\text { No. }=\mathbf{2 0}\end{array}$} & DM group & \multirow{2}{*}{ P-value } \\
\hline & & & & & No. $=20$ & \\
\hline \multirow{2}{*}{ 2D IVSd } & Mean \pm SD & \multirow{2}{*}{\multicolumn{2}{|c|}{$\begin{array}{l}9.21 \pm 0.85 \\
7.5-10.8\end{array}$}} & $12.07 \pm 0.25$ & $11.98 \pm 0.26$ & \multirow{2}{*}{0.000} \\
\hline & Range & & & $11.7-12.6$ & $11.5-12.4$ & \\
\hline \multirow{2}{*}{ 2D PWd } & Mean \pm SD & \multicolumn{2}{|c|}{$8.95 \pm 0.69$} & $11.45 \pm 0.48$ & $11.29 \pm 0.51$ & \multirow{2}{*}{0.000} \\
\hline & Range & \multicolumn{2}{|c|}{$8-10$} & $10.3-12$ & $10.2-12.3$ & \\
\hline \multirow{2}{*}{ 2D LVEF } & Mean \pm SD & \multirow{2}{*}{\multicolumn{2}{|c|}{$\frac{65.5 \pm 2.7}{62-70}$}} & $64.20 \pm 2.46$ & $64.50 \pm 2.54$ & \multirow{2}{*}{0.255} \\
\hline & Range & & & $60-68$ & $60-69$ & \\
\hline \multirow{2}{*}{ 2D EDV } & Mean \pm SD & \multirow{2}{*}{\multicolumn{2}{|c|}{$\frac{100.25 \pm 7.4}{88-112}$}} & $101.45 \pm 7.52$ & $104.10 \pm 5.38$ & \multirow{2}{*}{0.199} \\
\hline & Range & & & $89-112$ & $93-112$ & \\
\hline \multirow{2}{*}{ 2D ESV } & Mean \pm SD & \multicolumn{2}{|c|}{$34.85 \pm 8.15$} & $36.20 \pm 8.59$ & $38.70 \pm 4.90$ & \multirow{2}{*}{0.256} \\
\hline & Range & \multicolumn{2}{|c|}{$22-52$} & $23-51$ & $30-46$ & \\
\hline \multirow{2}{*}{ 2D SV } & Mean \pm SD & \multicolumn{2}{|c|}{$65.4 \pm 4.47$} & $65.25 \pm 2.99$ & $65.40 \pm 3.44$ & \multirow{2}{*}{0.989} \\
\hline & Range & \multicolumn{2}{|c|}{$57-72$} & $59-70$ & $58-72$ & \\
\hline \multirow{2}{*}{ 2D LV mass } & Mean \pm SD & $93.11 \pm$ & & $115.91 \pm 10.63$ & $114.19 \pm 8.83$ & 0 \\
\hline & Range & $83.6-1$ & & $97.4-131.7$ & $100.8-132.6$ & \\
\hline & Mean \pm SD & $95.63 \pm$ & & $120.05 \pm 10.80$ & $118.79 \pm 10.61$ & \\
\hline ASE & Range & $81.3-1$ & & $101.5-133.9$ & $100.8-133.7$ & \\
\hline & & & & th hoc analysis & & \\
\hline Variables & Control Vs HT & $\mathbf{N}$ group & & itrol Vs DM gro & HTN V & M group \\
\hline 2D IVSd & 0.000 & & & 0.000 & & \\
\hline 2D PWd & 0.000 & & & 0.000 & & 76 \\
\hline 2D LV mass & 0.000 & & & 0.000 & & \\
\hline ASE & 0.000 & & & 0.000 & & 18 \\
\hline
\end{tabular}

There was no statistically significant difference between the three groups and 3D echocardiographic data regarding 3D LVEF, 3D EDV, 3D ESV, 3D SV, while there was highly statistically significant difference as regard 3D IVSd, 3D PWd, 3D LV mass (Table 2). 
Table (1): Comparison between the studied groups regarding the 3D echocardiographic data

\begin{tabular}{|c|c|c|c|c|c|c|}
\hline \multirow{2}{*}{\multicolumn{2}{|c|}{ Parameters $\quad$ Groups }} & \multirow{2}{*}{\multicolumn{2}{|c|}{$\begin{array}{c}\text { Control group } \\
\text { No. }=\mathbf{2 0}\end{array}$}} & HTN group & DM group & \multirow{2}{*}{ P-value } \\
\hline & & & & No. $=20$ & No. $=20$ & \\
\hline \multirow{2}{*}{ 3D IVSd } & Mean \pm SD & \multirow{2}{*}{\multicolumn{2}{|c|}{$\frac{9.21 \pm 0.85}{7.5-10.8}$}} & $12.07 \pm 0.25$ & $11.98 \pm 0.26$ & \multirow{2}{*}{0.000} \\
\hline & Range & & & $11.7-12.6$ & $11.5-12.4$ & \\
\hline \multirow{2}{*}{ 3D PWd } & Mean \pm SD & \multicolumn{2}{|c|}{$8.95 \pm 0.69$} & $11.45 \pm 0.48$ & $11.29 \pm 0.51$ & \multirow{2}{*}{0.000} \\
\hline & Range & \multicolumn{2}{|c|}{$8-10$} & $10.3-12$ & $10.2-12.3$ & \\
\hline \multirow{2}{*}{ 3D LVEF } & Mean \pm SD & 66.42 & 2.32 & $65.29 \pm 2.44$ & $65.72 \pm 2.25$ & \multirow{2}{*}{0.314} \\
\hline & Range & 62.6 & 70.1 & $62.1-70.4$ & $61.4-69.3$ & \\
\hline \multirow{2}{*}{ 3D EDV } & Mean \pm SD & 130.6 & 10.9 & $129.70 \pm 6.97$ & $133.85 \pm 4.72$ & \multirow{2}{*}{0.230} \\
\hline & Range & $98-$ & & $117-141$ & $125-140$ & \\
\hline \multirow{2}{*}{ 3D ESV } & Mean \pm SD & 48.3 & 82 & $49.40 \pm 5.56$ & $45.00 \pm 5.43$ & \multirow[b]{2}{*}{0.061} \\
\hline & Range & & & $39-58$ & 54 & \\
\hline \multirow{2}{*}{ 3D SV } & Mean \pm SD & 64.25 & 4.46 & $64.40 \pm 3.38$ & $64.45 \pm 3.12$ & \multirow{2}{*}{0.984} \\
\hline & Range & $56-$ & & $59-70$ & $58-69$ & \\
\hline \multirow{2}{*}{ 3D LV mass } & Mean \pm SD & 93.18 & 12.8 & $123.04 \pm 10.63$ & $121.48 \pm 11.64$ & \multirow{2}{*}{0.000} \\
\hline & Range & $75.7-$ & 140.6 & $102.7-136.6$ & $100.9-136.5$ & \\
\hline \multirow{2}{*}{ Variables } & \multicolumn{6}{|c|}{ Post hoc analysis } \\
\hline & \multicolumn{2}{|c|}{ Control Vs HTN group } & \multicolumn{2}{|c|}{ Control Vs DM group } & \multicolumn{2}{|c|}{ HTN Vs DM group } \\
\hline 3D IVSd & \multicolumn{2}{|l|}{0.000} & \multicolumn{2}{|c|}{0.000} & \multicolumn{2}{|c|}{0.616} \\
\hline 3D P & \multicolumn{2}{|l|}{0.000} & & 0.000 & \multicolumn{2}{|c|}{0.376} \\
\hline 3D LV mass & \multicolumn{2}{|l|}{0.000} & & 0.000 & \multicolumn{2}{|c|}{0.676} \\
\hline
\end{tabular}

There was no statistically significant difference between the three groups and CMR data regarding CMR LVEF, CMR ESV, CMR SV, while there was highly statistically significant difference as regard CMR IVSd, CMR PWd, CMR EDV, CMR LV mass (Table 3). 
HOSSAM FARID HAMADA et al.,

Table (2): Comparison between the studied groups regarding the CMR data

\begin{tabular}{|c|c|c|c|c|c|c|c|}
\hline \multirow{2}{*}{\multicolumn{2}{|c|}{$\begin{array}{ll}\text { Parameters } & \text { Groups } \\
\end{array}$}} & \multirow{2}{*}{\multicolumn{2}{|c|}{$\begin{array}{c}\text { Control group } \\
\text { No. }=20 \\
\end{array}$}} & HTN group & \multirow{2}{*}{\multicolumn{2}{|c|}{$\begin{array}{c}\text { DM group } \\
\text { No. }=\mathbf{2 0}\end{array}$}} & \multirow{2}{*}{ P-value } \\
\hline & & & & No. $=20$ & & & \\
\hline \multirow{2}{*}{ CMR IVSd } & Mean \pm SD & \multicolumn{2}{|c|}{$9.17 \pm 0.72$} & $12.11 \pm 0.35$ & \multicolumn{2}{|c|}{$12.34 \pm 0.27$} & \multirow{2}{*}{0.000} \\
\hline & Range & \multicolumn{2}{|c|}{$7.6-10.7$} & $11.3-13.1$ & \multicolumn{2}{|c|}{$11.8-12.9$} & \\
\hline \multirow{2}{*}{ CMR PWd } & Mean \pm SD & \multicolumn{2}{|c|}{$9.03 \pm 0.7$} & $11.31 \pm 0.45$ & \multicolumn{2}{|c|}{$11.64 \pm 0.42$} & \multirow{2}{*}{0.000} \\
\hline & Range & \multicolumn{2}{|c|}{$7.6-10.4$} & $10.5-12.1$ & \multicolumn{2}{|c|}{$10.9-12.4$} & \\
\hline \multirow{2}{*}{ CMR LVEF } & Mean \pm SD & \multicolumn{2}{|c|}{$65.42 \pm 3.79$} & $65.97 \pm 2.84$ & \multicolumn{2}{|c|}{$65.85 \pm 2.72$} & \multirow{2}{*}{0.846} \\
\hline & Range & \multicolumn{2}{|c|}{$57.9-72.2$} & $58.6-71$ & \multicolumn{2}{|c|}{$58.8-71.5$} & \\
\hline \multirow{2}{*}{ CMR ESV } & Mean \pm SD & \multicolumn{2}{|c|}{$55.9 \pm 7.61$} & $51.39 \pm 5.43$ & \multicolumn{2}{|c|}{$53.58 \pm 5.83$} & \multirow{2}{*}{0.089} \\
\hline & Range & \multicolumn{2}{|c|}{$41-65.9$} & $42.4-59.8$ & \multicolumn{2}{|c|}{$43.3-62.4$} & \\
\hline \multirow{2}{*}{ CMR EDV } & Mean \pm SD & \multirow{2}{*}{\multicolumn{2}{|c|}{$\begin{array}{l}132.99 \pm 8.28 \\
112.4-143.2\end{array}$}} & $134.87 \pm 5.43$ & \multirow{2}{*}{\multicolumn{2}{|c|}{$\begin{array}{l}120.95 \pm 9.14 \\
107.5-134.6\end{array}$}} & \multirow{2}{*}{0.000} \\
\hline & Range & & & $122.6-143$ & & & \\
\hline \multirow{2}{*}{ CMR SV } & Mean \pm SD & \multicolumn{2}{|c|}{$67.18 \pm 10.45$} & $66.04 \pm 5.13$ & 67. & $1 \pm 4.27$ & \\
\hline & Range & $48.7-$ & 8.7 & $53.8-78.3$ & & -75.3 & \\
\hline & Mean \pm SD & $98.6 \pm 1$ & 5.31 & $130.79 \pm 8.95$ & 125. & $0 \pm 10.12$ & \\
\hline CM & Range & $83-1$ & & $112.7-148$ & & -146 & \\
\hline & & & & & & & \\
\hline$\sqrt{2}$ & Control Vs I & group & & Vs DM g & & HTN & Ig \\
\hline CMR IVSd & 0.000 & & & 0.000 & & & \\
\hline CMR PWd & 0.000 & & & 0.000 & & & 57 \\
\hline CMR EDV & 0.447 & & & 0.000 & & & 00 \\
\hline CMR LV mass & 0.000 & & & 0.000 & & & 78 \\
\hline
\end{tabular}

There was no statistically significant difference between the three measurements in control group data regarding IVSd, PWd, LVEF, SV, while there was highly statistically significant difference as regard ESV, EDV, LV mass (Table 4). 
Table (3): Pairwise comparison among the control group regarding the 2D, $3 \mathrm{D}$ and CMR measurements

\begin{tabular}{|c|c|c|c|c|c|}
\hline \multicolumn{2}{|c|}{\begin{tabular}{|ll} 
Parameters & Control group \\
\end{tabular}} & 2D & 3D & CMR & P-value \\
\hline \multirow{2}{*}{ IVSd } & Mean \pm SD & $9.21 \pm 0.85$ & $9.21 \pm 0.85$ & $9.17 \pm 0.72$ & \multirow{2}{*}{0.687} \\
\hline & Range & $7.5-10.8$ & $7.5-10.8$ & $7.6-10.7$ & \\
\hline \multirow{2}{*}{ PWd } & Mean \pm SD & $8.95 \pm 0.69$ & $8.95 \pm 0.69$ & $9.03 \pm 0.7$ & \multirow[b]{2}{*}{0.347} \\
\hline & Range & $8-10$ & $8-10$ & $7.6-10.4$ & \\
\hline \multirow{2}{*}{ LVEF } & Mean \pm SD & $65.5 \pm 2.7$ & $66.42 \pm 2.32$ & $65.42 \pm 3.79$ & \multirow{2}{*}{0.435} \\
\hline & Range & $62-70$ & $62.6-70.1$ & $57.9-72.2$ & \\
\hline \multirow{2}{*}{ ESV } & Mean \pm SD & $34.85 \pm 8.15$ & $48.3 \pm 6.82$ & $55.9 \pm 7.61$ & \multirow{2}{*}{0.000} \\
\hline & Range & $22-52$ & $31-57$ & $41-65.9$ & \\
\hline \multirow{2}{*}{ EDV } & Mean \pm SD & $100.25 \pm 7.4$ & $130.6 \pm 10.9$ & $132.99 \pm 8.28$ & \multirow{2}{*}{0.000} \\
\hline & Range & $88-112$ & $98-142$ & $112.4-143.2$ & \\
\hline \multirow{2}{*}{ SV } & Mean \pm SD & $65.4 \pm 4.47$ & $64.25 \pm 4.46$ & $67.18 \pm 10.45$ & \multirow{2}{*}{0.312} \\
\hline & Range & $57-72$ & $56-72$ & $48.7-88.7$ & \\
\hline \multirow{2}{*}{ LV mass } & Mean \pm SD & $93.11 \pm 10.91$ & $93.18 \pm 12.8$ & $98.6 \pm 15.31$ & \multirow{2}{*}{0.000} \\
\hline & Range & $83.6-134.5$ & $75.7-140.6$ & $83-156$ & \\
\hline \multirow{2}{*}{ Variables } & \multicolumn{5}{|c|}{ Post hoc analysis } \\
\hline & \multicolumn{3}{|l|}{$2 \mathrm{D} \mathrm{Vs} \mathrm{3D}$} & \multicolumn{2}{|c|}{ 3D Vs CMR } \\
\hline ESV & \multicolumn{2}{|l|}{0.000} & 0.000 & \multicolumn{2}{|c|}{0.000} \\
\hline EDV & \multicolumn{2}{|l|}{0.000} & 0.000 & \multicolumn{2}{|c|}{1.000} \\
\hline LV mass & \multicolumn{2}{|l|}{1.000} & 0.001 & \multicolumn{2}{|c|}{0.000} \\
\hline
\end{tabular}

There was no statistically significant difference between the measurements among the HTN group using 2D and 3D echocardiography and CMR regarding IVSd, SV, and there was statistically significant difference regarding PWd, LVEF, while there was highly statistically significant difference as regard ESV, EDV, LV mass (Table 5). 
HOSSAM FARID HAMADA et al.,

Table (4): Pairwise comparison among the hypertension group regarding the 2D, 3D and CMR measurements

\begin{tabular}{|c|c|c|c|c|c|}
\hline \multicolumn{2}{|c|}{$\begin{array}{ll}\text { HTN group } \\
\text { Parameters }\end{array}$} & 2D & 3D & CMR & P-value \\
\hline \multirow{2}{*}{ IVSd } & Mean \pm SD & $12.07 \pm 0.25$ & $12.07 \pm 0.25$ & $12.11 \pm 0.35$ & \multirow{2}{*}{0.759} \\
\hline & Range & $11.7-12.6$ & $11.7-12.6$ & $11.3-13.1$ & \\
\hline \multirow{2}{*}{ PWd } & Mean \pm SD & $11.45 \pm 0.48$ & $11.45 \pm 0.48$ & $11.31 \pm 0.45$ & \multirow{2}{*}{0.042} \\
\hline & Range & $10.3-12$ & $10.3-12$ & $10.5-12.1$ & \\
\hline \multirow{2}{*}{ LVEF } & Mean \pm SD & $64.20 \pm 2.46$ & $65.29 \pm 2.44$ & $65.97 \pm 2.84$ & \multirow{2}{*}{0.026} \\
\hline & Range & $60-68$ & $62.1-70.4$ & $58.6-71$ & \\
\hline \multirow{2}{*}{ ESV } & Mean \pm SD & $36.20 \pm 8.59$ & $49.40 \pm 5.56$ & $51.39 \pm 5.43$ & \multirow{2}{*}{0.000} \\
\hline & Range & $23-51$ & $39-58$ & $42.4-59.8$ & \\
\hline \multirow{2}{*}{ EDV } & Mean \pm SD & $101.45 \pm 7.52$ & $129.70 \pm 6.97$ & $134.87 \pm 5.43$ & \multirow{2}{*}{0.000} \\
\hline & Range & $89-112$ & $117-141$ & $122.6-143$ & \\
\hline \multirow{2}{*}{ SV } & Mean \pm SD & $65.25 \pm 2.99$ & $64.40 \pm 3.38$ & $66.04 \pm 5.13$ & \multirow{2}{*}{0.410} \\
\hline & Range & $59-70$ & $59-70$ & $53.8-78.3$ & \\
\hline \multirow{2}{*}{ LV mass } & Mean \pm SD & $115.91 \pm 10.63$ & $123.04 \pm 10.63$ & $130.79 \pm 8.95$ & \multirow{2}{*}{0.000} \\
\hline & Range & $97.4-131.7$ & $102.7-136.6$ & $112.7-148$ & \\
\hline \multirow{2}{*}{ Variables } & \multicolumn{5}{|c|}{ Post hoc analysis } \\
\hline & \multicolumn{2}{|c|}{ 2D Vs 3D } & D Vs CMR & \multicolumn{2}{|c|}{ 3D Vs CMR } \\
\hline LVEF & \multirow{2}{*}{\multicolumn{2}{|c|}{0.007}} & 0.052 & \multicolumn{2}{|c|}{0.903} \\
\hline ESV & & & 0.000 & \multicolumn{2}{|c|}{1.000} \\
\hline EDV & \multicolumn{2}{|l|}{$\begin{array}{l}0.000 \\
0.000\end{array}$} & 0.000 & \multicolumn{2}{|c|}{0.037} \\
\hline LV mass & \multicolumn{2}{|l|}{0.000} & 0.000 & 0.00 & \\
\hline
\end{tabular}

There was no statistically significant difference between DM group regarding the 2D and 3D echocardiography and CMR data regarding LVEF, SV, while there was highly statistically significant difference as regard IVSd, PWd, ESV, EDV, LV mass (Table 6). 
Table (5): Pairwise comparison among the diabetes group regarding the 2D, $3 \mathrm{D}$ and CMR measurements

\begin{tabular}{|c|c|c|c|c|c|}
\hline \multicolumn{2}{|c|}{ Parameters $\quad$ DM group } & 2D & 3D & CMR & P-value \\
\hline \multirow{2}{*}{ IVSd } & Mean \pm SD & \multirow{2}{*}{$\begin{array}{c}11.98 \pm 0.26 \\
11.5-12.4\end{array}$} & $11.98 \pm 0.26$ & $12.34 \pm 0.27$ & \multirow{2}{*}{0.000} \\
\hline & Range & & $11.5-12.4$ & $11.8-12.9$ & \\
\hline \multirow{2}{*}{ PWd } & Mean \pm SD & \multirow{2}{*}{$\frac{11.29 \pm 0.51}{10.2-12.3}$} & $11.29 \pm 0.51$ & $11.64 \pm 0.42$ & \multirow{2}{*}{0.000} \\
\hline & Range & & $10.2-12.3$ & $10.9-12.4$ & \\
\hline \multirow{2}{*}{ LVEF } & Mean \pm SD & $64.50 \pm 2.54$ & $65.72 \pm 2.25$ & $65.85 \pm 2.72$ & \multirow{2}{*}{0.109} \\
\hline & Range & $60-69$ & $61.4-69.3$ & $58.8-71.5$ & \\
\hline \multirow{2}{*}{ ESV } & Mean \pm SD & $38.70 \pm 4.90$ & $45.00 \pm 5.43$ & $53.58 \pm 5.83$ & \multirow{2}{*}{0.000} \\
\hline & Range & $30-46$ & $32-54$ & $43.3-62.4$ & \\
\hline \multirow{2}{*}{ EDV } & Mean \pm SD & $104.10 \pm 5.38$ & $133.85 \pm 4.72$ & $120.95 \pm 9.14$ & \multirow{2}{*}{0.000} \\
\hline & Range & $93-112$ & $125-140$ & $107.5-134.6$ & \\
\hline \multirow{2}{*}{ SV } & Mean \pm SD & $65.40 \pm 3.44$ & $64.45 \pm 3.12$ & $67.21 \pm 4.27$ & \multirow{2}{*}{0.076} \\
\hline & Range & $58-72$ & $58-69$ & $58.6-75.3$ & \\
\hline \multirow{2}{*}{ LV mass } & Mean \pm SD & $114.19 \pm 8.83$ & $121.48 \pm 11.64$ & $125.70 \pm 10.12$ & \multirow{2}{*}{0.000} \\
\hline & Range & $100.8-132.6$ & $100.9-136.5$ & $108-146$ & \\
\hline \multirow{2}{*}{ Variables } & \multicolumn{5}{|c|}{ Post hoc analysis } \\
\hline & \multicolumn{2}{|l|}{ 2D Vs 3D } & Vs CMR & \multicolumn{2}{|c|}{ 3D Vs CMR } \\
\hline IVSd & \multicolumn{2}{|l|}{1.000} & 0.000 & \multicolumn{2}{|c|}{0.000} \\
\hline PWd & \multicolumn{2}{|l|}{1.000} & 0.000 & \multicolumn{2}{|c|}{0.000} \\
\hline ESV & \multicolumn{2}{|l|}{0.003} & 0.000 & \multicolumn{2}{|c|}{0.000} \\
\hline EDV & \multicolumn{2}{|l|}{0.000} & 0.000 & \multicolumn{2}{|c|}{0.000} \\
\hline
\end{tabular}

\section{DISCUSSION}

LVM is strongly influenced by body size. However, even after adjustment for anthropometric variables, males have larger LVM than females (Marwick et al., 2015). Similarly, athletes have increased LVM compared to non-athletes (Poppe et al., 2015), and black men and women have larger LVM than their white or Asian counterparts (Lang et al., 2015). Likewise, obesity is associated with increased LVM. The aforementioned body size- , ethnic-, and exercise-related factors are associated with increased LVM, as well as proportional increases in left ventricular (LV) volume, which initially maintains normal LV wall stress (Poppe et al., 2015). Consequently, LV relative wall thickness (RWT), defined as the ratio of twice the LV infero-lateral wall thickness to the LV internal diameter measured at end-diastole, initially remains unchanged. Other factors to be considered are age and blood pressure.

Normal values for LVM are derived from studies of the general population without hypertension or obesity (Chirinos et al., 2010). Separate cutoff values for body size-adjusted LVM have been used for men and women (Su et al., 2012). In order to allow comparison of LVM among subjects of different body sizes, different allometric approaches have been suggested to normalize LVM (Chirinos et al., 2010). However, there is controversy about the best method for indexing LVM.

Body surface area (BSA) was the first anthropometric variable used to index LVM and has shown a stronger statistical correlation than height with LVM and 


\section{HOSSAM FARID HAMADA $e t$ al.,}

better identification of hypertensionrelated LVH. However, indexing by BSA has been noted to minimize the effect of obesity on LVM, and, therefore, it underestimates the prevalence of obesityrelated LVH. Consequently, height has also been used for indexing (either height alone or height raised to an allometric power of 1.7 or 2.7. Indexation of LVM to height raised to an allometric exponent of 2.7 (LVM/height2.7), in comparison to BSA or height alone, has shown better predictive value for CVD outcomes, better detection of obesity-related LVH, and less variability of LVM among normal individuals. Chirinos et al. demonstrated that indexation to $\mathrm{LVM}$ /height1.7 was the best method, in comparison to BSA and height 2.7, to identify obesity-related LVH and was more consistently associated with CVD outcomes and all-cause mortality (Chirinos et al., 2010). In a population with a low prevalence of obesity, there was no significant difference in the risk attributed to $\mathrm{LVH}$ regardless of the method of indexation. BSA has been widely adopted by the American Society of Echocardiography (ASE) and European Association of Cardiovascular Imaging as the preferred method for indexing LVM (Chirinos et al., 2010).

There is evidence suggesting that type II DM could be associated with increased LV mass, concentric geometry/remodeling and impaired diastolic function. Type II DM can cause LVH through metabolic and not hemodynamic pathways ( $\mathrm{Su}$ et al., 2012). Over time, these structural and functional changes result in impaired systolic function and symptomatic heart failure, which are associated with worse clinical outcomes.
The presence of LVH is a strong independent predictor of cardiovascular morbidity and mortality. Direct cardiac effects of LVH include increased risk for developing congestive heart failure, an increased risk of arrhythmic events and a reduced coronary flow reserve promoting myocardial ischemic episodes. In $\mathrm{LVH}$ there is an association between cerebrovascular disease and increased LV mass.

Different cardiac modalities are used for the evaluation of cardiac function, among these techniques, Echocardiography is still the commonly used in clinical practice thanks to its simple protocol and availability. In the past years, several studies have shown that the functional features computed from echocardiography are influenced by numerous factors such as heart rate and reduced image quality (Chahal et al., 2015). Moreover, the main limitation of echocardiography lies in the fact that it is an operator-dependent technique, which may lead to inter and intra-observer variability as well as to measurement errors.

While echocardiography remains the most used technique in daily cardiology practice, CMRI allows an accurate global and regional assessment of cardiac wall motion abnormalities and provides additional information regarding cardiac structure and function. For the computation of global LV features using MRI, myocardial contours' (endocardial and epicardial contours) delineation through a stack of cine MRI images in short axis view is needed. Then, the software available in the acquisition console can provide the following 
parameters: $\mathrm{CO}, \mathrm{EDV}, \mathrm{ESV}, \mathrm{SV}$, and LVEF.

Despite the diversity of techniques dedicated to the measurement of cardiac parameters and the increased number of research in this field, there is no consensus about the most reproducible and accurate method for the measurement of $\mathrm{LV}$ volumes and LVEF. Some studies have established a comparison of LV volumes and LVEF measurements using MRI, 2D and 3D echocardiography. Most of them have demonstrated that CMRI technique is more reproducible than 2D echocardiography since it is able to compute these functional parameters without geometric assumption and it is less dependent from the operator. In addition, they reported that the measurements obtained by 3D echo are similar to those obtained by MRI with little variation (Augustine et al., 2018).

CMR tissue tagging allowed the noninvasive assessment of intra-myocardial displacement / strain by monitoring motion of specific material points spread in the myocardium (Bratincsák et al., 2015). The application of this technique in large epidemiologic studies such as the Multi-Ethnic Study of Atherosclerosis (MESA) (Bacharova et al., 2015), has enabled to investigate the nature of atherosclerosis in a total of 1184 asymptomatic participants (aged 45-84 years). Regional LV function was quantified by evaluating peak systolic circumferential strain (Ecc). The study proved that higher diastolic blood pressure (DBP) was associated with decreased regional LV function in asymptomatic individuals and was significantly attenuated after controlling for LVM.
Furthermore, LV torsional deformation was greater in hypertensive patients, despite that they had lower circumferential shortening, because torsion in hypertension with concentric remodeling is a compensatory mechanism to maintain LVEF.

In our study, patients with type II DM and no hypertension, free of ischemic heart disease, had significantly larger LVMI in comparison to patients with both type II DM and hypertension and those with only hypertension. In the real clinical setting, patients with type II DM usually have co-existing hypertension, both known to contribute to the increase of the LVMI. Therefore, we expected to find the highest LVMI in that group, which was not the case. HTN per se is a well-known cause of LV mass increased (Levelt et al., 2016). However our study investigated patients with only hypertension and had smallest LVMI. Our results were in contrast to the strong heart study which showed that the combination of type II DM and HTN lead to the highest LVMI, followed by patients with only type II DM or HTN. Largest LVMI in the type II DM group indicates the possible negative effect of hyperglycemia on LV mass increase, even before overt type II DM.

The present study demonstrated that both 2D and 3D echocardiography allowed the estimation of LV EF. However, evaluation with 2D echo and 3D Echo results in significant underestimation of LV EF. In our study, reliability of $\mathrm{LV} \mathrm{EF}$ and $\mathrm{LV}$ measurements was superior with $3 \mathrm{D}$ echo. As compared with CMR, ECHO systematically overestimates LVM. 
Underestimation of LVM was seen in another study involving healthy subjects.

A major contributor to the inaccuracy in LVM measurement by ECHO relates to the presupposition of an ellipsoid shaped heart. The endocardial border may not be well defined on ECHO, and left ventricular wall thickness is not uniform across all myocardial segments. Furthermore, the ECHO calculation for LVM is based on a formula that relies heavily on the internal diameter of the left ventricle. As a result, LVM determined by ECHO can vary considerably, because of fluctuations in intravascular volume and the resulting change in intracardiac volume (Augustine et al., 2018). By contrast, CMR measures LVM via direct mathematical integration using 3dimensional data that does not involve assumptions regarding cardiac geometry or reliance on left ventricular diameter (Chirinos et al., 2010). CMR, unlike ECHO, is therefore less sensitive to changes in intra-vascular and by extension intra-cardiac volume. The limitations of 2dimensional ECHO may be mitigated by the use of 3-dimensional ECHO, which correlates better with CMR.

Our study expanded on previous work by evaluating paired ECHO and CMR studies in a comparator group, the exclusion of papillary muscle mass measurement from LVM determination by CMR may have inflated the mean difference in LVM by ECHO and CMR. However, given that ECHO-based LVM measurements universally exclude papillary muscles, a direct comparison excluding papillary muscles from the CMR protocol is actually more appropriate. Nevertheless, we tried to account for this by performing a sensitivity analysis in which LVM values were adjusted with the best estimate available for papillary muscle.

Despite its limitations, ECHO is advantageous in terms of accessibility and cost. Nevertheless, when it comes to the measurement of LVM and assessment of $\mathrm{LVH}$, the use of ECHO may overestimate the prevalence of $\mathrm{LVH}$.

By contrast, CMR which is less sensitive to volume changes provides a more accurate LVM measurement, in recognition of this major drawback of ECHO, clinical trials in which LVM is used as an outcome measure are increasingly relying on CMR. Hence, it is clear that CMR is beginning to emerge as the reference standard in research, particularly in those with left ventricular alterations that may not conform to ECHO geometric assumptions. This study also provides further support for the use of CMR in clinical practice when accurate measurements of LVM are needed.

Our findings demonstrated a significant degree of LVM overestimation and increased LVH prevalence by ECHO in patients with type II DM compared with normal individuals, while ECHO continued to be used in routine clinical practice, evidence was mounting that CMR may afford a more accurate evaluation of cardiac remodeling and associated LVM changes in type II DM patients, thus providing a superior alternative for cardiac assessment in this population.

\section{CONCLUSION}

In the assessment of LVM, no superiority between echocardiography and 
CMR may be stated at this time due to the absence of studies directly comparing the methods. Assessed by both echocardiography and CMR, LVM and LVH are reliable cardiovascular event predictors. LVM assessed by echocardiography is more practical on a clinical basis. CMR would be preferable for research and specific clinical conditions requiring higher accuracy and reproducibility.

Although detailed guidelines determining the clinical indications of CMR in hypertension are still missing, CMR can provide early and highly reproducible evaluation of $\mathrm{LVH}$ and remodeling, not available by any other non-invasive technique; furthermore, the capability to perform tissue characterization facilitates the early diagnosis and better risk stratification of micro-, macro-vascular ischemia and fibrosis, commonly found in hypertensive patients, with potentially high impact on their treatment and also on health care costs.

\section{REFERENCES}

1. Ambale-Venkatesh B, Volpe GJ, Donekal S, Mewton N, Liu CY, Shea S, Liu K, Burke G, Wu C, Bluemke DA and Lima JA (2014): Association of longitudinal changes in left ventricular structure and function with myocardial fibrosis: the multi-ethnic study of atherosclerosis study. Hypertension, 64:508-515.

2. American Society of Echocardiography Recommendations for Cardiac Chamber Quantification in Adults (2017): A Quick Reference Guide from the ASE Workflow and Lab Management Task Force
3. Augustine, D.X. and Howard L. (2018): Left ventricular Hypertrophy in Athletes: Differentiating Physiology from Pathology. Curr Treat Options Cardio Med, 10.1007/s11936-018-0691-2

4. Aurich M, André F, Keller M, Greiner S, Hess A, Buss SJ, Katus HA and Mereles D (2014): Assessment of left ventricular volumes with echocardiography and cardiac magnetic resonance imaging: real-life evaluation of standard versus new semiautomatic methods. Journal of the American Society of Echocardiography, 27(10):1017-1024.

5. Bacharova L, Chen H, Estes EH, Mateasik A, Bluemke DA, Lima JA, Burke GL and Soliman EZ (2015): Determinants of discrepancies in detection and comparison of the prognostic significance of left ventricular hypertrophy by electrocardiogram and cardiac magnetic resonance imaging. The American Journal of Cardiology, 115(4):515-522.

6. Bratincsák A, Williams M, Kimata $\mathrm{C}$ and Perry JC (2015): The electrocardiogram is a poor diagnostic tool to detect left ventricular hypertrophy in children: a comparison with echocardiographic assessment of left ventricular mass. Congenital Heart Disease, 10(4):E164-71.

7. Brouwer WP, van Dijk SJ, Stienen GJ, van Rossum $A C$, van der Velden $J$ and Germans T (2011): The development of familial hypertrophic cardiomyopathy: from mutation to bedside. European Journal of Clinical Investigation, 41(5):568-78.

8. Brumback LC, Kronmal R, Heckbert SR, Ni H, Hundley WG, Lima JA and Bluemke DA (2010): Body size adjustments for left ventricular mass by cardiovascular magnetic resonance and their impact on left ventricular hypertrophy 
HOSSAM FARID HAMADA et al.,

classification. The International Journal of Cardiovascular Imaging, 26(4):459-68.

9. Chahal H, Bluemke DA, Wu CO, McClelland R, Liu K, Shea SJ, Burke G, Balfour P, Herrington D, Shi Pand Post W (2015): Heart failure risk prediction in the Multi-Ethnic Study of Atherosclerosis. Heart, 101(1):58-64.

10. Chirinos JA, Segers P, De Buyzere ML, Kronmal RA, Raja MW, De Bacquer D, Claessens T, Gillebert TC, John-Sutton $M$ and Rietzschel ER (2010): Left ventricular mass: allometric scaling, normative values, effect of obesity, and prognostic performance. Hypertension, 56(1):91-8.

11.Drazner MH (2011): The progression of hypertensive heart disease. Circulation, 123(3):327-334.

12.Kusunose K, Kwon DH, Motoki H, Flamm SD and Marwick TH (2013): Comparison of three-dimensional echocardiographic findings to those of magnetic resonance imaging for determination of left ventricular mass in patients with ischemic and non-ischemic cardiomyopathy. The American Journal of Cardiology, 112(4):604-11.

13.Lang RM, Badano LP, Mor-Avi V, Afilalo J, Armstrong A, Ernande L, Flachskampf FA, Foster E, Goldstein SA, Kuznetsova $T$ and Lancellotti $P$ (2015): Recommendations for cardiac chamber quantification by echocardiography in adults: an update from the American Society of Echocardiography and the European Association of Cardiovascular Imaging. European Heart Journal-Cardiovascular Imaging, 16(3):233-71.

14.Levelt E, Mahmod M, Piechnik SK, Ariga R, Francis JM, Rodgers CT, Clarke WT, Sabharwal N, Schneider JE, Karamitsos TD and Clarke K (2016): Relationship between left ventricular structural and metabolic remodeling in type 2 diabetes. Diabetes, 65(1):44-52.

15.Liu CY, Liu YC, Wu C, Armstrong A, Volpe GJ, Van der Geest RJ, Liu Y, Hundley WG, Gomes AS, Liu S and Nacif M (2013): Evaluation of age-related interstitial myocardial fibrosis with cardiac magnetic resonance contrast-enhanced T1 mapping: MESA (Multi-Ethnic Study of Atherosclerosis). Journal of the American College of Cardiology, 62(14):1280-7.

16.Marwick TH, Gillebert TC, Aurigemma G, Chirinos J, Derumeaux G, Galderisi M, Gottdiener J, Haluska B, Ofili E, Segers $P$ and Senior $R$ (2015): Recommendations on the use of echocardiography in adult hypertension: a report from the European Association of Cardiovascular Imaging (EACVI) and the American Society of Echocardiography (ASE). European Heart JournalCardiovascular Imaging, 16(6):577-605.

17.Park JH, Negishi K, Kwon DH, Popovic ZB, Grimm RA and Marwick TH (2014): Validation of global longitudinal strain and strain rate as reliable markers of right ventricular dysfunction: comparison with cardiac magnetic resonance and outcome. Journal of Cardiovascular Ultrasound, 22(3):113.

18.Poppe KK, Doughty RN, Gardin JM, Hobbs FD, McMurray JJ, Nagueh SF, Senior R, Thomas L, Whalley GA, Aune E and Brown A (2015): On behalf of the Echocardiographic Normal Ranges MetaAnalysis of the Left Heart Collaboration. Ethnic-Specific Normative Reference Values for Echocardiographic LA and LV Size, LV Mass, and Systolic Function: The EchoNoRMAL Study. JACC Cardiovasc Imaging, 8:656-65.

19.Schelbert EB, Cao JJ, Sigurdsson S, Aspelund T, Kellman P, Aletras AH, Dyke CK, Thorgeirsson G, Eiriksdottir G, Launer LJ and Gudnason V (2012): 
Prevalence and prognosis of unrecognized myocardial infarction determined by cardiac magnetic resonance in older adults. JAMA, 308(9):890-6.

20.Su HM, Lin TH, Hsu PC, Chu CY, Lee WH, Chen SC, Lee CS, Voon WC, Lai WT and Sheu SH (2012): Association of interarm systolic blood pressure difference with atherosclerosis and left ventricular hypertrophy. PloS one, 7(8):e41173.

21.Turkbey EB, Nacif MS, Guo M, McClelland RL, Teixeira PB, Bild DE, Barr RG, Shea S, Post W, Burke G and Budoff MJ (2015): Prevalence and correlates of myocardial scar in a US cohort. JAMA, 314(18):1945-54.

22.Westenberg JJ, Braun J, Van de Veire NR, Klautz RJ, Versteegh MI, Roes SD, van der Geest RJ, de Roos A, van der Wall EE, Reiber JH and Bax JJ (2010): Magnetic resonance imaging assessment of reverse left ventricular remodeling late after restrictive mitral annuloplasty in early stages of dilated cardiomyopathy. The Journal of Thoracic and Cardiovascular Surgery, 135(6):1247-53.

23.Yim D, Nagata H, Lam CZ, GrosseWortmann L, Seed M, Jaeggi $E$ and Yoo SJ (2018): Disharmonious patterns of heterotaxy and isomerism: how often are the classic patterns breached?. Circulation: Cardiovascular Imaging, 11(2):e006917.

24.Yoneyama K, Gjesdal O, Choi EY, Wu CO, Hundley WG, Gomes AS, Liu CY, McClelland RL, Bluemke DA and Lima JA (2012): Age, sex, and hypertensionrelated remodeling influences left ventricular torsion assessed by tagged cardiac magnetic resonance in asymptomatic individuals: the multi-ethnic study of atherosclerosis. Circulation, 126(21):2481-90. 


\section{قيمة الموجات فوق الصوتية للقلب في تقييم تضخم البطين الأيسر في

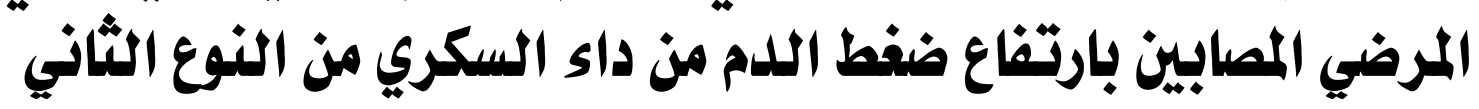
حسام فريد محمد أحمد حمادة، أحمد كمال مطاوع، محمد سعد الجمال، محمد اسماعيل

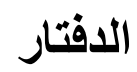

قسم امراض القلب، كلية الطب (القاهرة)، جامعة الازهر

E-mail: hossamfarid@hotmail.com

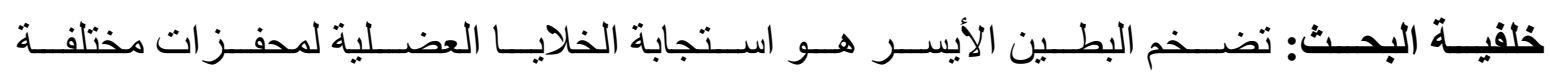

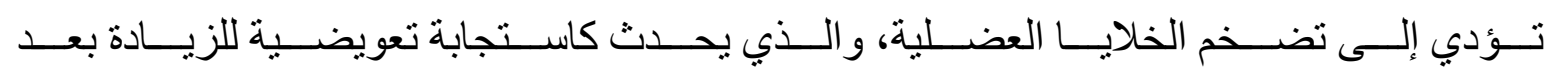

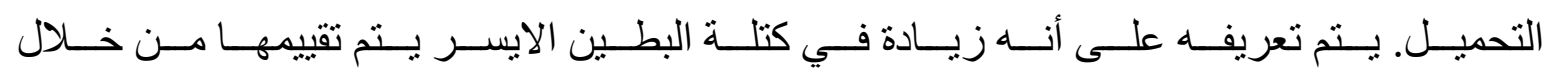

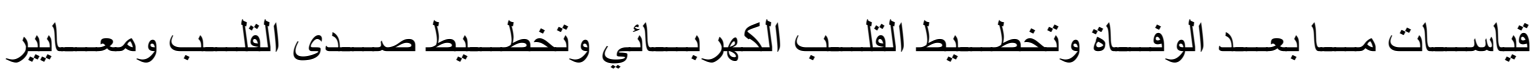
الرنين المغناطيسي للقلب و الأو عية الدموية.

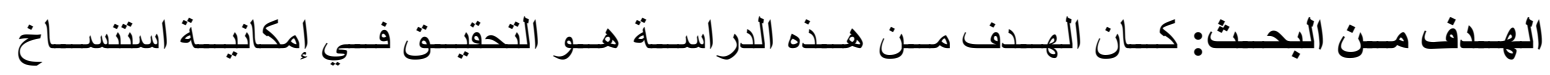

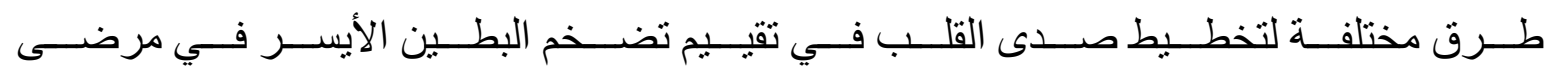

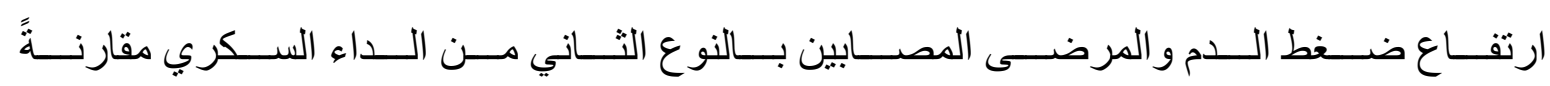
بالقياسات القياسية الذهبية للرنين المغناطيسي للقلب.

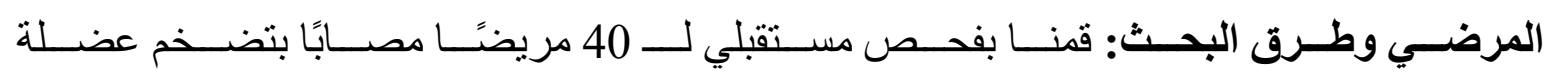

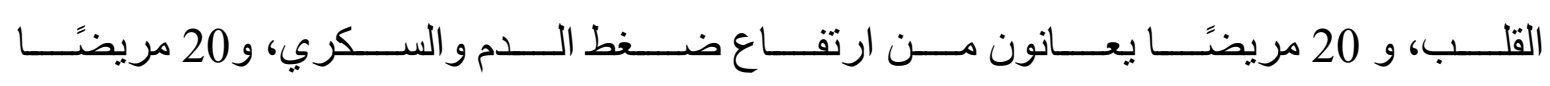

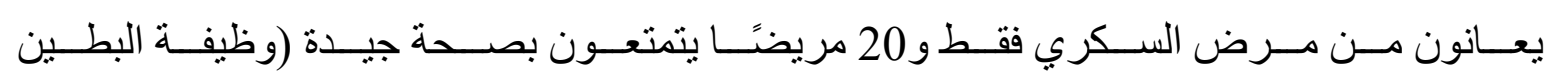

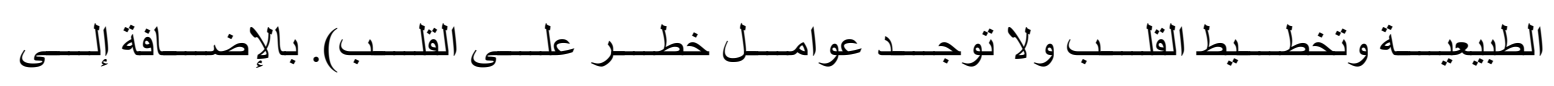

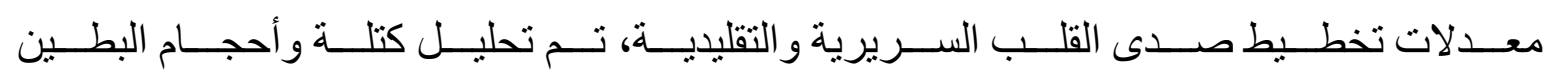

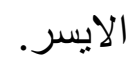

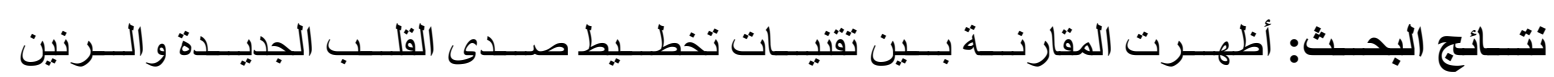

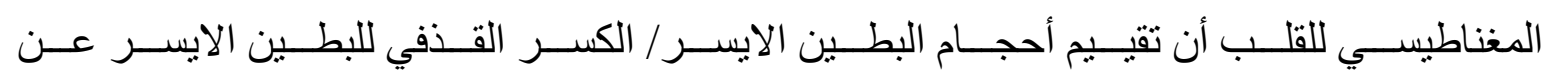

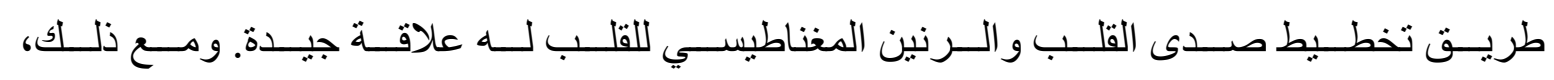

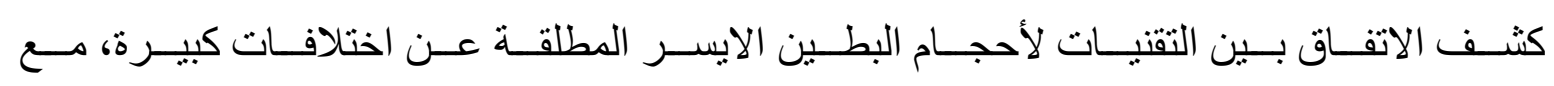

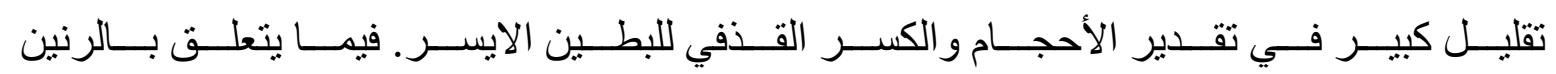




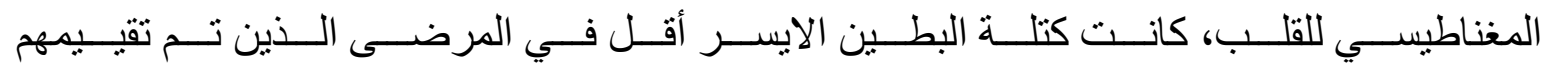

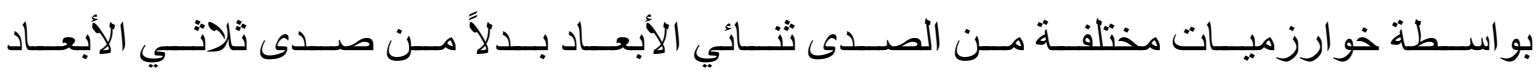

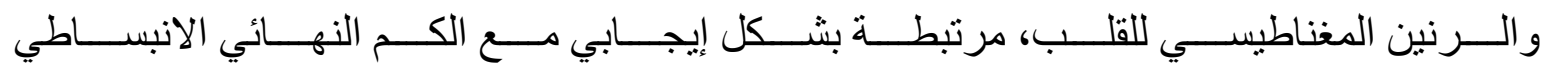
و الكم النهائي الانقباضي.

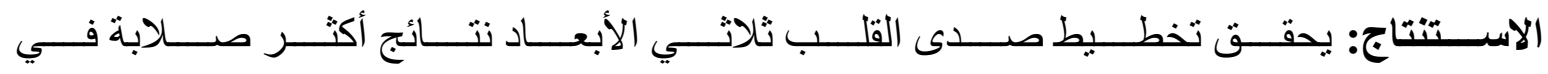

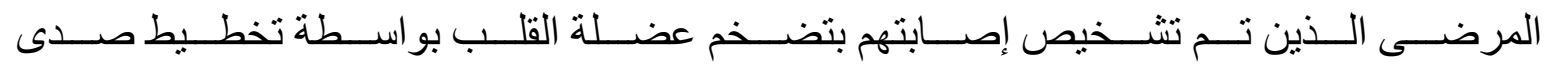

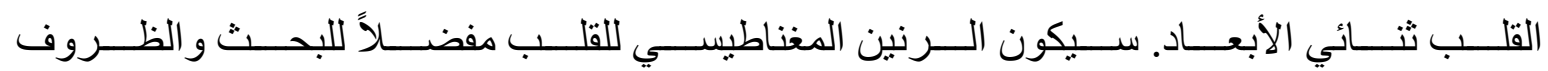
السريرية المحددة التي تتطلاب دقة أعلى.

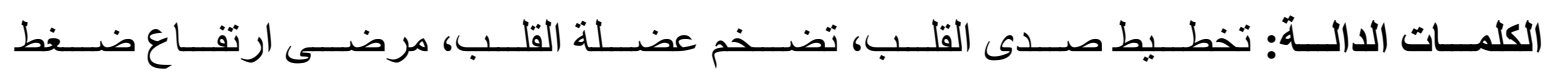
الدم، داء السكري من النوع الثاني. 